\title{
Superluminal propagation via coherent manipulation of the Raman gain process
}

\author{
G. S. Agarwal and Shubhrangshu Dasgupta \\ Physical Research Laboratory, Navrangpura, Ahmedabad 380 009, India
}

(Received 8 February 2004; published 12 August 2004)

\begin{abstract}
We present a method of manipulating the Raman process by using a coherent control field which leads to splitting of the Raman gain peak into a doublet and anomalous dispersion in the region between the gain peaks. We show how the region of almost no Raman gain and strong anomalous dispersion is ideally suited for producing superluminal propagation. In particular, we show that the group index for a ${ }^{23} \mathrm{Na}$ condensate could be in the range $-10^{3}$ to $-10^{4}$.

DOI: $10.1103 /$ PhysRevA.70.023802

PACS number(s): 42.50.Gy, 42.65.Dr
\end{abstract}

It is now widely recognized that the dispersive properties of a medium can be well controlled by the application of a coherent field on an accompanying unoccupied transition $[1,2]$. This type of dispersion management has led to interesting applications in nonlinear optics and in the propagation of pulses. Harris et al. recognized the possibility of ultraslow light [3], which was realized by Hau et al. [4]. There have been many other realizations of ultraslow light in atomic vapors [5] and solid state materials [6]. The work on ultraslow light has further led to proposals on the stopping of light [7] which has been achieved in very recent experiments [8]. Fiberlike dispersion in an atomic vapor can be produced [9]. We have also seen parallel developments in superluminal propagation. Based on the earlier suggestion of Steinberg and Chiao [10], Wang et al. produced a double-peaked Raman gain profile using two pump fields with closely separated frequencies [11]. They obtained superluminal propagation of pulses with central frequency near the minimum in the double-peaked Raman gain profile. Bigelow et al. [12] realized superluminal propagation in alexandrite by using population pulsations and reverse saturation in this medium. Propagation of pulses with discontinuities has been studied with a view to obtaining the correct signal velocities [13]. There are many other theoretical proposals for achieving superluminality. Agarwal et al. [14] suggested the use of a microwave field between the two lower levels of a $\Lambda$ system. Cross talk between different fields can also lead to superluminality [15]. Note that the cross talk is especially important when the separation between the two lower levels is of the order of or less than the Rabi frequency of the pump field. More specifically, Wilson-Gordon and co-workers [16] have shown that Zeeman systems reverse dispersion with increase of the strength of the pumping field.

In this paper, we propose a scheme in which Raman gain processes can be coherently controlled and show how this coherent control can lead to large superluminality in a fashion similar to the realization of ultraslow light using coherent control. It is known that the dispersion on the probe transition which corresponds to Raman gain should be normal in the region of the line center. For large superluminality we need to create anomalous dispersion with very little gain so that pulses can propagate without growing or becoming unstable. This indeed is produced by the application of a coherent control field which creates a hole in the gain profile in the region of the line center. This is reminiscent of the hole in the absorption profile produced by a coherent field [17]. Thus, by the application of a coherent field, we produce a sharp minimum in the Raman gain profile and a steep anomalous dispersion. This coupled with a large density provides us with ideal conditions for superluminal propagation of light pulses. It must be noted that the control of other two-photon processes has been discussed. For example, electromagnetically induced transparency in two-photon absorption has been proposed [18] and seen [19,20].

Our model for coherent control of Raman gain processes is shown schematically in Fig. 1. The ground levels $|g\rangle$ and $\left|g^{\prime}\right\rangle$ are coupled to the excited level $|e\rangle$ by electric fields with Rabi frequencies $G_{1}$ and $G_{2}$, respectively. The corresponding detunings are $\Delta_{1}$ and $\delta$, respectively, defined as the differences between the frequencies of the interacting fields and the corresponding transition frequencies. The control field is applied on the transition $\left|g^{\prime}\right\rangle$ and $\left|e^{\prime}\right\rangle$ and will enable us to manipulate the Raman gain [21]. We note that the Raman gain is a single-step process where the pumping field $G_{1}$ is detuned from the transition. We will continue to work under detuned conditions, otherwise there would be a mixing of the two-step $\left(|g\rangle \rightarrow|e\rangle \rightarrow\left|g^{\prime}\right\rangle\right)$ and one-step processes. Since the systems used in many specific applications of coherent control appear rather similar, it is good to point out the major differences. In the case of ultraslow light we have a weak probe pulse whose propagation in a linear medium is controlled by a coherent field. The probe pulse acts on a transi-

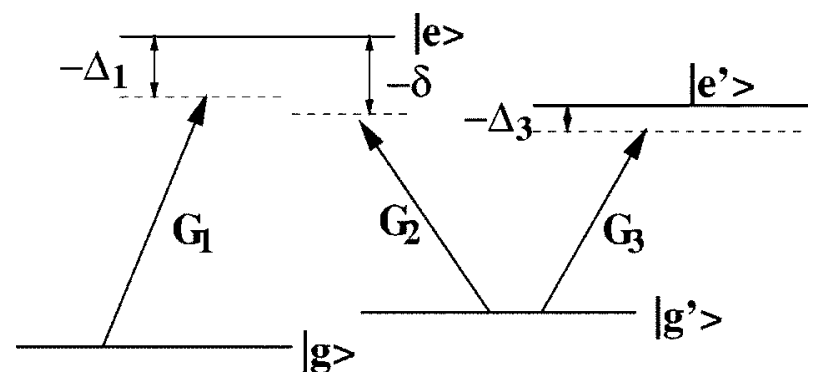

FIG. 1. Level configuration for achieving superluminality. The pump and probe fields with respective Rabi frequencies $G_{i}(i$ $\in 1,2)$ interact with the transitions $|e\rangle \leftrightarrow|g\rangle$ and $|e\rangle \leftrightarrow\left|g^{\prime}\right\rangle$, respectively. Superluminality can be created by applying a control field with Rabi frequency $G_{3}$ that couples the level $\left|e^{\prime}\right\rangle$ to $\left|g^{\prime}\right\rangle$. $\Delta_{1}, \delta$, and $\Delta_{3}$ are the respective detunings. 

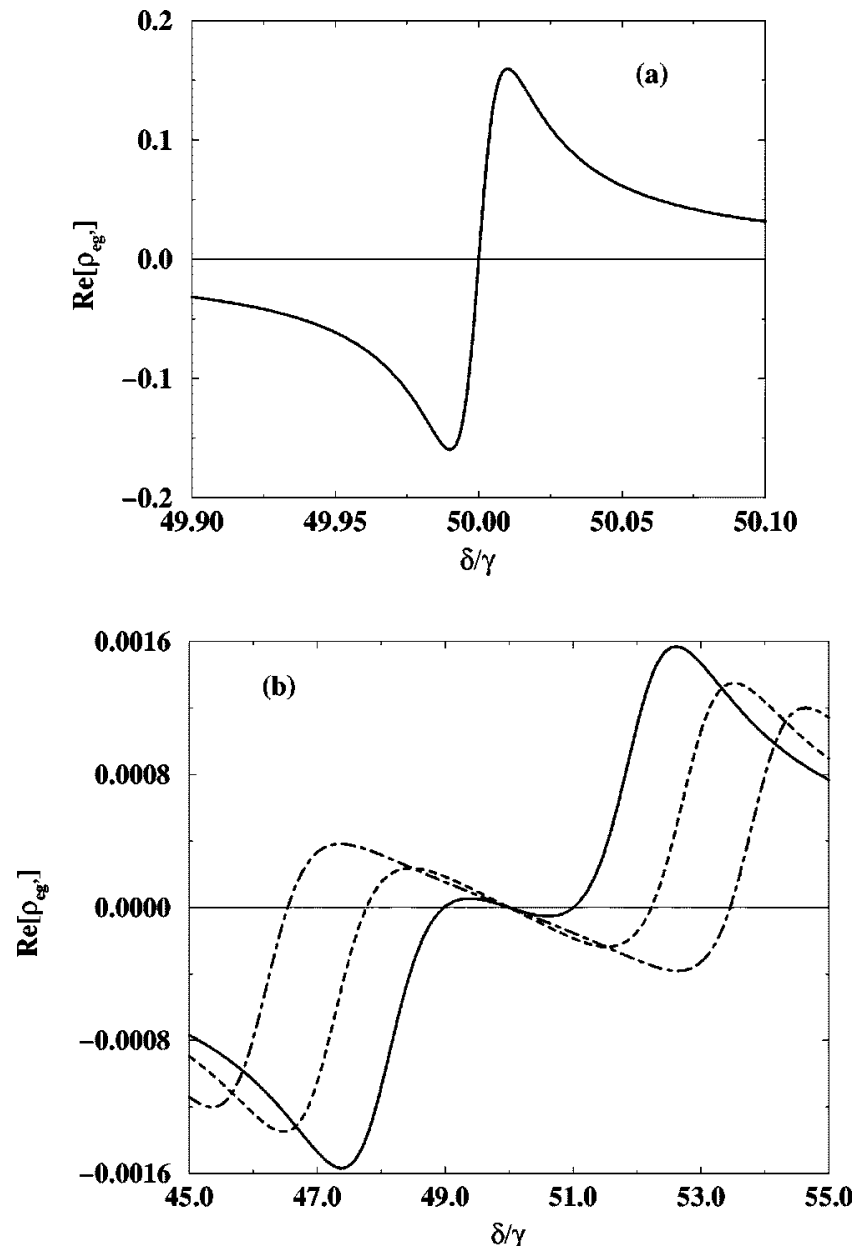

FIG. 2. Variation of the real part $\rho_{e g^{\prime}}$ with probe detuning $\delta$. The specific parameters chosen here are (a) $G_{3}=0$, (b) $G_{3}=4.5 \gamma$ (solid line), $G_{3}=6 \gamma$ (dashed line), and $G_{3}=8 \gamma$ (dot-dashed line). The other parameters used here are $\Delta_{1}=50 \gamma, \Delta_{3}=0, G_{1}=G_{2}=4 \gamma, \gamma_{g e}=\gamma_{g^{\prime} e}$ $=\gamma_{g^{\prime} e^{\prime}}=\gamma_{g e^{\prime}}=2 \gamma, \Gamma_{g g^{\prime}}=0.01 \gamma$ (which accounts for the collisional relaxation rate), $\Gamma_{e^{\prime} g^{\prime}}=\Gamma_{e^{\prime} g}=\Gamma_{e g^{\prime}}=\Gamma_{e g}=2.01 \gamma$, and $\Gamma_{e e^{\prime}}=4.01 \gamma$.

tion with population in the lower state. In a Raman gain process the probe pulse acts between levels that are not normally populated and there is a pump on a transition that has population in the lower state. The Raman gain process itself is a second-order process. In addition, we apply a coherent field to control the second-order Raman process.

By incorporating the spontaneous decay rates $\gamma_{l e}$ and $\gamma_{l e^{\prime}}$ $\left(l \in g, g^{\prime}\right)$ from the levels $|e\rangle$ and $\left|e^{\prime}\right\rangle$ to the ground levels, we perform full density matrix calculations using the equations given in the Appendix for the four-level system to second order in the pump field and to first order in the probe field. This is the condition under which Raman gain processes are normally studied. The relevant part of the polarization (the density matrix element) for the single-step Raman process is found to be

$$
\begin{aligned}
\rho_{e g^{\prime}} \equiv & \frac{G_{1}^{2} G_{2}}{8}\left[\frac{-2 i \Gamma_{e g}}{\left(\gamma_{g e}+\gamma_{g^{\prime} e}\right)\left(\Gamma_{e g}^{2}+\Delta_{1}^{2}\right)\left(\Gamma_{e g^{\prime}}-i \delta\right)}\right. \\
& \left.+\frac{-i}{\left(\Gamma_{e g}+i \Delta_{1}\right)\left\{\Gamma_{g g^{\prime}}-i\left(\delta-\Delta_{1}\right)\right\}\left(\Gamma_{e g^{\prime}}-i \delta\right)}\right],
\end{aligned}
$$

where $\Gamma_{\alpha \beta}$ is the dephasing rate of the coherence between the levels $|\alpha\rangle$ and $|\beta\rangle$. Note the presence of the Raman resonance in the second term in the above equation. Under the conditions in which the Raman gain is normally studied, the term without the Raman resonant term is unimportant. Thus in the limit of large detuning $\delta \sim \Delta_{1} \gg \gamma$ 's, we get approximately

$$
\rho_{e g^{\prime}} \equiv \frac{-i G_{1}^{2} G_{2}}{8 \delta \Delta_{1}\left\{\Gamma_{g g^{\prime}}-i\left(\delta-\Delta_{1}\right)\right\}} .
$$

The real and imaginary parts of Eq. (1) give the dispersion and the Raman gain $\left[\operatorname{Im}\left(\rho_{e g^{\prime}}\right)<0\right]$. Note that the Raman dispersion is normal in the region of Raman resonance [see Fig. 2(a)]. As mentioned earlier, we need to obtain a region of almost no gain with, however, steep anomalous dispersion. This can be achieved by the control laser on the transition $\left|e^{\prime}\right\rangle \leftrightarrow\left|g^{\prime}\right\rangle$. Using the full density matrix equations and keeping terms of all orders in the control field, we find the result for the nonlinear Raman susceptibility $\chi$ which now depends on the control field:

$$
\chi=\frac{2 N\left|\vec{d}_{e g^{\prime}}\right|^{2}}{\hbar G_{2}} \rho_{e g^{\prime}}=\frac{3 N \lambda^{3}}{32 \pi^{3}} G_{1}^{2} \mathcal{D}
$$

where

$$
\mathcal{D}=\frac{-i}{A}\left[\frac{2 \Gamma_{e g}\left\{\Gamma_{e e^{\prime}}-i\left(\delta-\Delta_{3}\right)\right\}}{\left(\gamma_{g e}+\gamma_{g^{\prime} e}\right)\left(\Gamma_{e g}^{2}+\Delta_{1}^{2}\right)}+\frac{\left\{\Gamma_{e e^{\prime}}-i\left(\delta-\Delta_{3}\right)\right\}\left\{\Gamma_{e^{\prime} g}-i\left(\delta-\Delta_{1}-\Delta_{3}\right)\right\}-\left(\left|G_{3}\right|^{2} / 4\right)}{\left(\Gamma_{e g}+i \Delta_{1}\right)\left[\left\{\Gamma_{g g^{\prime}}-i\left(\delta-\Delta_{1}\right)\right\}\left\{\Gamma_{e^{\prime} g}-i\left(\delta-\Delta_{1}-\Delta_{3}\right)\right\}+\left(\left|G_{3}\right|^{2} / 4\right)\right]}\right]
$$

and $A=\left(\Gamma_{e g^{\prime}}-i \delta\right)\left\{\Gamma_{e e^{\prime}}-i\left(\delta-\Delta_{3}\right)\right\}+\left(\left|G_{3}\right|^{2} / 4\right)$. Here $\rho_{e g^{\prime}}$ $=\left(G_{1}^{2} G_{2}\right) \mathcal{D} / 8$ is the new optical coherence, modified by control field, $N$ is the number density of the atomic medium, $\lambda$ $=2 \pi c / \omega$ is the wavelength of the $|e\rangle \leftrightarrow\left|g^{\prime}\right\rangle$ transition, $\omega$ is the corresponding angular frequency, and $\left|\vec{d}_{e g^{\prime}}\right|$ is the magni- tude of the dipole matrix element between the levels $|e\rangle$ and $\left|g^{\prime}\right\rangle$. We have used the fact that the total decay rate $4 \gamma$ from the level $|e\rangle$ equals the Einstein coefficient $A$ $=4\left|\vec{d}_{e g^{\prime}}\right|^{2} \omega^{3} / 3 \hbar c^{3}$. Note that the Raman gain coefficient can be calculated from 

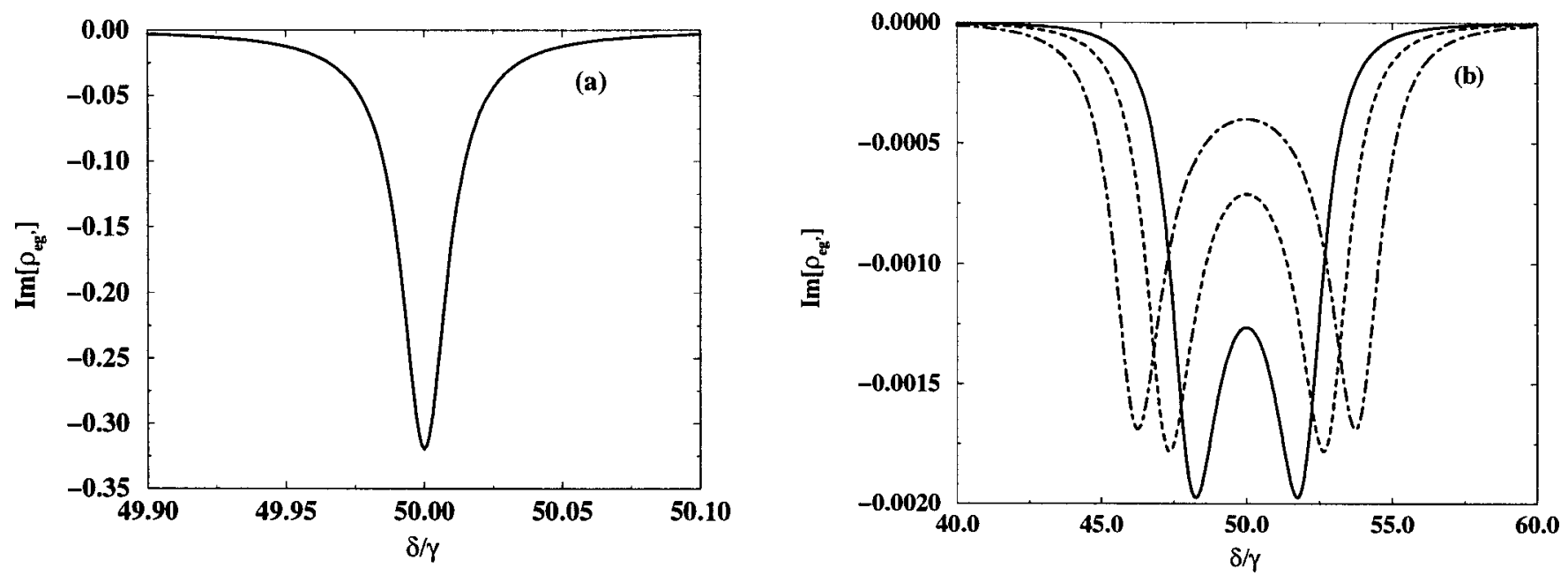

FIG. 3. Variation of the imaginary part of $\rho_{e g^{\prime}}$ with probe detuning $\delta$. The specific parameters chosen here are (a) $G_{3}=0,(\mathrm{~b}) G_{3}$ $=4.5 \gamma$ (Solid line), $G_{3}=6 \gamma$ (dashed line), and $G_{3}=8 \gamma$ (dot-dashed line). The other parameters are the same as in Fig. 2 .

$$
G_{R}=-4 \pi k \operatorname{Im}(\chi)=-\frac{3 N \lambda^{2}}{4 \pi} G_{1}^{2} \operatorname{Im} \mathcal{D},
$$

where $k=2 \pi / \lambda$ is the wave number of the interacting pulse and $N$ is the atomic number density.

We show the variation of the real and imaginary parts of the Raman susceptibility $\chi$ in units of $2 N\left|\vec{d}_{e g^{\prime}}\right|^{2} / \hbar G_{2}$ with the detuning $\delta$ of the probe field in Figs. 2 and 3. One clearly sees the Raman gain at $\delta=\Delta_{1}$ for $G_{3}=0$ [see Fig. 3(a)]. If now one applies a control field that is resonant with the corresponding transition $\left|e^{\prime}\right\rangle \leftrightarrow\left|g^{\prime}\right\rangle$, then the gain peak splits into a doublet. This is attributed to the fact that the resonant control field dresses the levels $\left|e^{\prime}\right\rangle$ and $\left|g^{\prime}\right\rangle$ into the superpositions $| \pm\rangle=\left(\left|e^{\prime}\right\rangle \pm\left|g^{\prime}\right\rangle\right) / \sqrt{2}$ of the states $\left|e^{\prime}\right\rangle,\left|g^{\prime}\right\rangle$. The two peaks refer to the Raman gain corresponding to the transitions $\left|e^{\prime}\right\rangle$ to $| \pm\rangle$.

As can be seen from Fig. 3(b), if $G_{3} \neq 0$, then at the twophoton resonance $\delta=\Delta_{1}$, the gain of the medium [ $\equiv$ imaginary part of $\chi$, Eq. (3)] is reduced by a large extent. Also, the nature of the dispersion of the medium $[\equiv$ real part of $\chi$, Eq. (3)] in the region of the frequencies of the probe field varies from normal to anomalous if one switches the control field on (see Fig. 2). The slope of the anomalous dispersion can be manipulated using the Rabi frequency $G_{3}$ of the control field.

Note that we could also directly integrate the complete set of density matrix equations and compute the real and imaginary parts of the susceptibilities. In this calculation, we have to choose the field strengths so that the perturbative results are recovered. In addition, we also have to choose the detuning $\Delta_{1}$ such that the two-step process is unimportant. Some typical results are shown in Fig. 4 where we show a comparison of the perturbative and numerical results. A small shift in the numerical results is to be noted. This arises due to the finiteness of the field strengths $G_{1}$ and $G_{2}$ relative to the detuning $\Delta_{1}$.

Having discussed the control of the Raman gain and the dispersion of the Raman susceptibility, we next examine the propagation of a pulse on the Raman transition $|e\rangle \leftrightarrow\left|g^{\prime}\right\rangle$. We assume that the central frequency of the pulse is given by
$\delta=\Delta_{1}$. The group velocity of the Raman pulse can be evaluated in terms of the Raman susceptibility through the relation

$$
v_{g}=\frac{c}{n_{g}}, \quad n_{g}=1+2 \pi \operatorname{Re}(\chi)+2 \pi \omega \frac{\partial}{\partial \omega} \operatorname{Re}(\chi) .
$$

Here the group index $n_{g}$ is to be calculated at $\delta=\Delta_{1}$.

Using the relations (3)-(6), we calculate the group index $n_{g}$ and Raman gain $G_{R} l$ at the Raman resonance $\delta=\Delta_{1}$, where $l$ is the length of the medium. We show their variation with the Rabi frequency $G_{3}$ of the resonant control field in Fig. 5 for propagation in a ${ }^{23} \mathrm{Na}$ condensate medium. Note that a negative value of the group index $n_{g}$ refers to the situation when the pulse advancement $\left(1-n_{g}\right) l / c$ becomes much larger than its transit time $l / c$ in vacuum. The Raman pulse is narrowed depending on the amount of Raman gain, which decreases on increasing the control field. Further, the

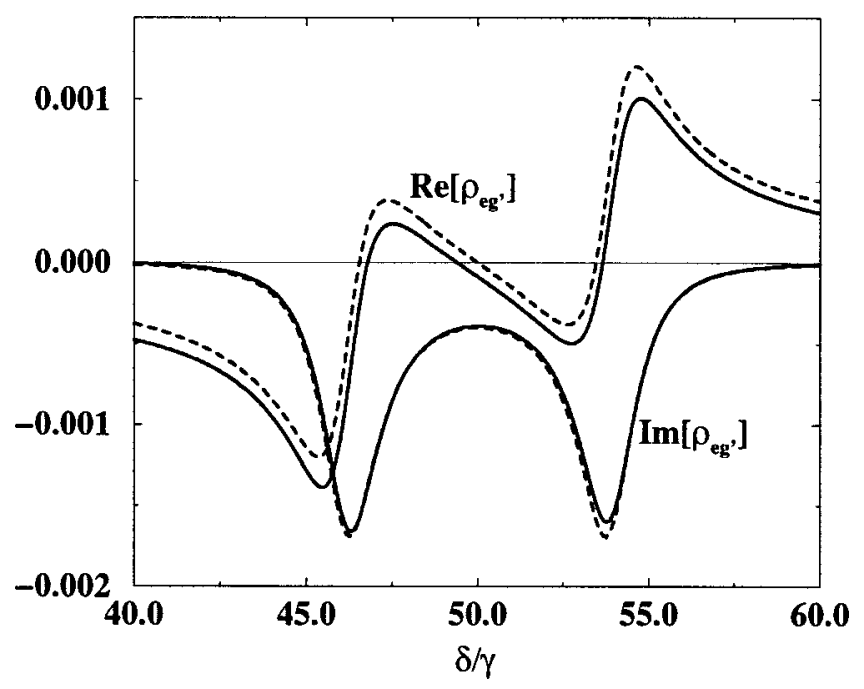

FIG. 4. Variation of real and imaginary parts of $\rho_{e g^{\prime}}$ calculated using the complete set of density matrix equations (solid lines) and using the perturbative solution [Eq. (4); dashed lines] for $G_{3}=8 \gamma$. The other parameters chosen here are the same as in Fig. 2. 


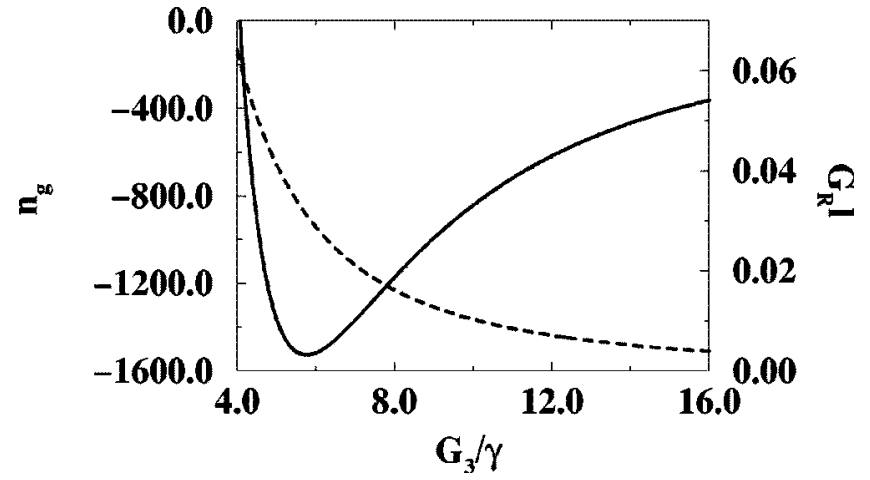

FIG. 5. Variation of the group index $n_{g}$ (solid line, scale on the left hand side) and total gain $G_{R} l$ (dashed line, scale on the right hand side) with the control field Rabi frequency $G_{3}$ for $\Delta_{3}=0$. We have assumed the parameters of a ${ }^{23} \mathrm{Na}$ condensate $[\lambda=589.6 \mathrm{~nm}$ for the $D_{1}$ transition $\left.\left(|e\rangle \leftrightarrow|g\rangle,\left|g^{\prime}\right\rangle\right)\right]$. Here the length $l$ of the medium is $50 \mu \mathrm{m}$ as in a Bose condensate, the number density $N=5$ $\times 10^{12} \mathrm{~cm}^{-3}$, and $G_{1}=G_{2}=4 \gamma$. The other parameters are $\Delta_{1}=50 \gamma$, $\gamma_{g e}=\gamma_{g^{\prime} e}=\gamma_{g^{\prime} e^{\prime}}=\gamma_{g e^{\prime}}=2 \gamma, \quad \Gamma_{g g^{\prime}}=0.01 \gamma, \quad \Gamma_{e^{\prime} g^{\prime}}=\Gamma_{e^{\prime} g}=\Gamma_{e g^{\prime}}=\Gamma_{e g}$ $=2.01 \gamma$, and $\Gamma_{e e^{\prime}}=4.01 \gamma$. Note that the Einstein A coefficient for the $D_{2}$ transition $\left(\left|e^{\prime}\right\rangle \leftrightarrow|g\rangle,\left|g^{\prime}\right\rangle\right)$ for ${ }^{23} \mathrm{Na}$ is $2 \pi \times 9.795 \times 10^{6} \mathrm{~s}^{-1}$.

group index can become as large as -1527 for a small value of $G_{3} \sim 5.76 \gamma$, which is larger than the one reported $(\sim-310)$ in Raman experiments in Cs vapor [11]. Note, however, that the pulse advancement for a group index of the order of -1000 for propagation over a distance of the order of $100 \mu \mathrm{m}$ will be $\sim 0.3 \mathrm{~ns}$. On the other hand, the gain of the pulse inside the medium remains of the order of $1.03\left(G_{R} l=0.0311\right.$ at $G_{3}=5.76 \gamma$ for $\left.l=50 \mu \mathrm{m}\right)$. We thus have the possibility of large superluminality using appropriate coherent control. Note further that the group index is sensitive to the value of $\gamma_{g^{\prime} e^{\prime}}$. For example, we obtain values of $n_{g}$ for $G_{3}=6 \gamma$ of -1518 for $\gamma_{g^{\prime} e^{\prime}}=\gamma_{g e^{\prime}}=2 \gamma$ and -1928 for $\gamma_{g^{\prime} e^{\prime}}=\gamma_{g e^{\prime}}=0.02 \gamma$. We further calculated the group index near two-photon resonance, where $\operatorname{Re}\left(\rho_{e g^{\prime}}\right)$ vanishes. For $G_{3}=8 \gamma$, this is equal to -1180 at $\delta=49.4 \gamma$, which is almost equal to that at two-photon resonance, $\delta=50 \gamma$.

The group index $n_{g}$ can be further increased if one increases the detuning $\Delta_{3}$ of the control field. The variation of $n_{g}$ with control field Rabi frequency $G_{3}$ for different values of $\Delta_{3}$ is shown in Fig. 6. Note that the group index can be made much larger -13836 for $\Delta_{3}=5 \gamma$, at smaller $G_{3}$ $\sim 4.09 \gamma$. We have seen that even for $G_{3}=5.76 \gamma$ the value of $n_{g}$ can be enhanced to as much as -7821 for $\Delta_{3}=5 \gamma$. The gain $G_{R} l$, however, does not change significantly for larger $\Delta_{3}$ for a moderate value of $G_{3}$. Only for larger $G_{3}$ can the gain in the medium become smaller.

It is possible to obtain a much simplified expression for $n_{g}$ for resonant control field strengths and detunings much larger than $\Gamma$ and for $\Gamma_{e^{\prime} g} \ll \gamma$ :

$$
\frac{\partial \chi}{\partial \delta} \approx-\frac{3 N \lambda^{3}}{32 \pi^{3}}\left(\frac{G_{1}^{2}}{\delta^{2}}\right) \frac{4}{G_{3}^{2}} .
$$

Its similarity to the result for the case of ultraslow light [4] is interesting. The minus sign yields superluminal propagation. The extra factor $\left(G_{1}^{2} / \delta^{2}\right)$ is a reflection of the fact that the

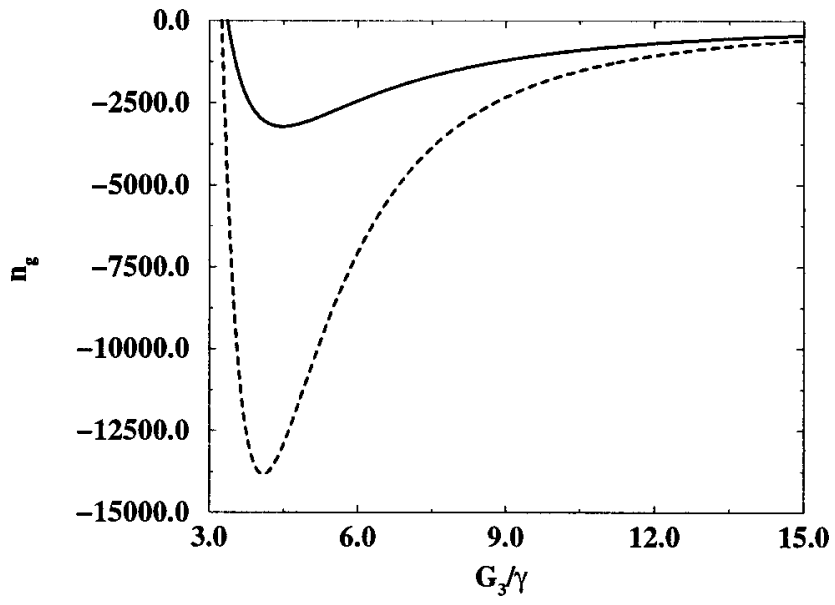

FIG. 6. Variation of the group index $n_{g}$ with the control field Rabi frequency $G_{3}$ for $\Delta_{3}=2 \gamma$ (solid line) and $\Delta_{3}=5 \gamma$ (dashed line). The other parameters are the same as in Fig. 5.

pump field has to create Raman coherence. We mention the main difference between the work of Wang et al. and ours. In our model, the control field creates two dressed states and the gain doublet arises as a consequence of Raman transitions to these states. On the other hand, in the work of Wang et al., the gain doublet is created by two different pump fields with closely separated frequencies, where there is no possibility of coherence-induced effects. Thus the susceptibility in the case of Refs. 10 and 11 can be written as a sum of two independent Lorentzians.

In conclusion, we have presented a model showing the possibility of superluminal propagation through coherent manipulation of a Raman process. This can be achieved by applying a control field so that the Raman gain peak splits into a doublet and thereby produces anomalous dispersion in the region between the two peaks. We further demonstrate the possibility of large superluminality in a Bose condensate. The achievable values are sensitive to the relaxation parameters and control field detuning.

\section{APPENDIX: WORKING EQUATIONS FOR COHERENT CONTROL OF THE RAMAN SYSTEM}

For the sake of completeness and notational clarity, we summarize the density matrix equations for the four-level system of Fig. 1. The basic equations under the rotating wave approximation are as follows:

$$
\begin{gathered}
\dot{\rho}_{e e}=\frac{i}{2}\left[G_{1} \rho_{g e}+G_{2} \rho_{g^{\prime} e}-\text { c.c. }\right]-\left(\gamma_{g e}+\gamma_{g^{\prime} e}\right) \rho_{e e}, \\
\dot{\rho}_{e^{\prime} e^{\prime}}=\frac{i}{2}\left[G_{3} \rho_{g^{\prime} e^{\prime}}-\text { c.c. }\right]-\left(\gamma_{g^{\prime} e^{\prime}}+\gamma_{g e^{\prime}}\right) \rho_{e^{\prime} e^{\prime}}, \\
\dot{\rho}_{g g}=\frac{i}{2}\left[G_{1}^{*} \rho_{e g}-\text { c.c. }\right]+\gamma_{g e} \rho_{e e}+\gamma_{g e^{\prime}} \rho_{e e^{\prime}}, \\
\dot{\rho}_{e^{\prime} g^{\prime}}=\left[i \Delta_{3}-\Gamma_{e^{\prime} g^{\prime}}\right] \rho_{e^{\prime} g^{\prime}}+\frac{i}{2} G_{3}\left[\rho_{g^{\prime} g^{\prime}}-\rho_{e^{\prime} e^{\prime}}\right]-\frac{i}{2} G_{2} \rho_{e^{\prime} e},
\end{gathered}
$$




$$
\begin{gathered}
\dot{\rho}_{e g^{\prime}}=\left[i \delta-\Gamma_{e g^{\prime}}\right] \rho_{e g^{\prime}}+\frac{i}{2} G_{2}\left[\rho_{g^{\prime} g^{\prime}}-\rho_{e e}\right]+\frac{i}{2} G_{1} \rho_{g g^{\prime}} \\
-\frac{i}{2} G_{3} \rho_{e e^{\prime}}, \\
\dot{\rho}_{e g}=\left[i \Delta_{1}-\Gamma_{e g}\right] \rho_{e g}+\frac{i}{2} G_{1}\left[\rho_{g g}-\rho_{e e}\right]+\frac{i}{2} G_{2} \rho_{g^{\prime} g}, \\
\dot{\rho}_{g g^{\prime}}=\left[i\left(\delta-\Delta_{1}\right)-\Gamma_{g g^{\prime}}\right] \rho_{g g^{\prime}}+\frac{i}{2} G_{1}^{*} \rho_{e g^{\prime}}-\frac{i}{2} G_{2} \rho_{g e}-\frac{i}{2} G_{3} \rho_{g e^{\prime}}, \\
\dot{\rho}_{e e^{\prime}}=\left[i\left(\delta-\Delta_{3}\right)-\Gamma_{e e^{\prime}}\right] \rho_{e e^{\prime}}+\frac{i}{2} G_{1} \rho_{g e^{\prime}}+\frac{i}{2} G_{2} \rho_{g^{\prime} e^{\prime}}-\frac{i}{2} G_{3}^{*} \rho_{e g^{\prime}},
\end{gathered}
$$

$$
\dot{\rho}_{e^{\prime} g}=\left[i\left(\Delta_{1}+\Delta_{3}-\delta\right)-\Gamma_{e^{\prime} g}\right] \rho_{e^{\prime} g}+\frac{i}{2} G_{3} \rho_{g^{\prime} g}-\frac{i}{2} G_{1} \rho_{e^{\prime} e},
$$

where $\rho_{e e}+\rho_{g g}+\rho_{e^{\prime} e^{\prime}}+\rho_{g^{\prime} g^{\prime}}=1$. The actual values of the offdiagonal elements are the ones given by Eq. (A1) with additional time-dependent phases $\rho_{e^{\prime} g^{\prime}} e^{-i \omega_{3} t}, \rho_{e g^{\prime}} e^{-i \omega_{2} t}, \rho_{e g} e^{-i \omega_{1} t}$, $\rho_{g g^{\prime}} e^{i\left(\omega_{1}-\omega_{2}\right) t}, \rho_{e e^{\prime}} e^{i\left(\omega_{3}-\omega_{2}\right) t}$, and $\rho_{e^{\prime} g} e^{-i\left(\omega_{1}-\omega_{2}+\omega_{3}\right) t}$. Here, the detunings of the pump, probe, and control fields are $\Delta_{1}$ $=\omega_{1}-\omega_{e g}, \delta=\omega_{2}-\omega_{e g^{\prime}}$, and $\Delta_{3}=\omega_{3}-\omega_{e^{\prime} g^{\prime}}$, respectively, the $\omega_{i}$ 's $(i \in 1,2,3)$ being the respective angular frequencies. The Rabi frequencies of these fields are defined as $G_{1}$ $=2 \vec{d}_{e g} \cdot \vec{E}_{1} / \hbar, G_{2}=2 \vec{d}_{e g^{\prime}} \cdot \vec{E}_{2} / \hbar$, and $G_{3}=2 \vec{d}_{e^{\prime} g^{\prime}} \cdot \vec{E}_{3} / \hbar$, where the $E_{i}$ 's are the respective field amplitudes. The decay rate $\Gamma_{\alpha \beta}$ of the coherence between the levels $|\alpha\rangle$ and $|\beta\rangle$ is defined as $\Gamma_{\alpha \beta}=\sum_{i}\left(\gamma_{i \alpha}+\gamma_{i \beta}\right)+\gamma_{\text {coll }}$, where $\gamma_{\alpha \beta}$ is the spontaneous decay rate from the level $|\beta\rangle$ to $|\alpha\rangle$ and $\gamma_{\text {coll }}$ is the collisional decay rate.
[1] S. P. Tewari and G. S. Agarwal, Phys. Rev. Lett. 56, 1811 (1986).

[2] S. E. Harris, J. E. Field, and A. Imamoglu, Phys. Rev. Lett. 64, 1107 (1990);K. H. Hahn, D. A. King, and S. E. Harris, ibid. 65, 2777 (1990).

[3] S. E. Harris, J. E. Field, and A. Kasapi, Phys. Rev. A 46, R29 (1992).

[4] L. V. Hau et al., Nature (London) 397, 594 (1999).

[5] M. M. Kash et al., Phys. Rev. Lett. 82, 5229 (1999);D. Budker et al.,ibid. 83, 1767 (1999).

[6] A. V. Turukhin et al., Phys. Rev. Lett. 88, 023602 (2002).

[7] O. Kocharovskaya, Y. Rostovtsev, and M. O. Scully, Phys. Rev. Lett. 86, 628 (2001).

[8] M. Bajcsy, A. S. Zibrov, and M. D. Lukin, Nature (London) 426, 638 (2003).

[9] R. Kapoor and G. S. Agarwal, Phys. Rev. A 61, 053818 (2000).

[10] A. M. Steinberg and R. Y. Chiao, Phys. Rev. A 49, 2071 (1994).

[11] L. J. Wang, A. Kuzmich, and A. Dogariu, Nature (London) 406, 277 (2000);A. Dogariu, A. Kuzmich, and L. J. Wang, Phys. Rev. A 63, 053806 (2001).

[12] M. S. Bigelow, N. N. Lepeshkin, and R. W. Boyd, Phys. Rev. Lett. 90, 113903 (2003); Science 301, 200 (2003).
[13] M. D. Stenner, D. J. Gauthier, and M. A. Neifeld, Nature (London) 425, 695 (2003).

[14] G. S. Agarwal, T. N. Dey, and S. Menon, Phys. Rev. A 64, 053809 (2001).

[15] S. Menon and G. S. Agarwal, Phys. Rev. A 59, 740 (1999);S. Menon, Ph.D thesis, Mohanlal Sukhadia University, Udaipur, India, 2000, p. 46.

[16] A. D. Wilson-Gordon and H. Friedman, J. Mod. Opt. 49, 125 (2002);C. Goren, A. D. Wilson-Gordon, M. Rosenbluh, and H. Friedman, Phys. Rev. A 68, 043818 (2003).

[17] S. E. Harris, Phys. Today 50 (7), 46 (1997).

[18] G. S. Agarwal and W. Harshawardhan, Phys. Rev. Lett. 77, 1039 (1996).

[19] J.- Y. Gao et al., Phys. Rev. A 61, 023401 (2000);D. Wang et al., Europhys. Lett. 54, 456 (2001).

[20] M. Yan, E. G. Rickey, and Y. Zhu, Phys. Rev. A 64, 043807 (2001); Y. Wu, J. Saldana, and Y. Zhu, ibid. 67, 013811 (2003).

[21] The energy level scheme of Fig. 1 is similar to the one studied by S. E. Harris and Y. Yamamato [ Phys. Rev. Lett. 81, 3611 (1998); for experimental verification of this proposal, see M. Yan, E. G. Rickey, and Y. Zhu, Phys. Rev. A 64, 041801 (2001)] in connection with switching of the two-photon absorption. However, by applying the pump and probe on different transitions, we study the control of the Raman gain. 